\title{
Nonlinear absorption and dispersion in fiber coupled silicon photonic crystal microresonators
}

\author{
Paul E. Barclay, Kartik Srinivasan, and Oskar Painter \\ Thomas J. Watson, Sr: Laboratory of Applied Physics, California Institute of Technology, Pasadena, CA 91125, USA. \\ phone: (626) 395-6269, fax: (626) 795-7258, e-mail: pbarclay@caltech.edu
}

\begin{abstract}
Two-photon absorption, free-carrier absorption and dispersion, and thermo-optic dispersion in high-Q, ultra-small mode volume, photonic crystal cavities are studied experimentally. Sub-nanosecond free-carrier lifetimes, and optical bistablity at $\sim 100 \mu \mathrm{W}$ cavity input power are observed.

(c) 2005 Optical Society of America

OCIS codes: (190.1450) Bistability, (230.5750) Resonators
\end{abstract}

Resonant microresonators formed in planar photonic crystals are capable of confining light to ultra-small mode volumes for long cavity photon lifetimes[1,2], resulting in extremely large stored electromagnetic energy densities for modest input power. It has been anticipated [3, 4] that this local field enhancement will allow nonlinear optical effects in photonic crystal $(\mathrm{PC})$ cavities to be observable at $\sim \mathrm{mW}$ input powers. Recently, optical bistability in silicon ring resonators was used to demonstrate all-optical on-chip functionality [5, 6]. The increased field enhancement achievable in photonic crystal mirocavities promises to allow equivalent functionality to be demonstrated at lower input powers, and the sub-wavelength size of PC cavities suggests that they may support faster switching speeds. Similarily, PC cavities can support coherent interaction between a single emitter, for example a quantum dot [7,8] or a cold cesium atom [9], and a single intracavity photon, and promise the realization of on-chip cavity quantum electrodynamics experiments in the strong coupling regime. Here we investigate the nonlinear optical response of a high-Q PC cavity formed in silicon. We observe optical bistability for $100 \mu \mathrm{W}$ dropped cavity power, and predict sub-nanosecond free carrier lifetimes in the PC cavity. These measurements are enabled by an efficient fiber taper to PC cavity evanescent coupling channel [10], which is also of general use for studying, for example, future applications of PC cavities in quantum optics, where photon collection is an important measure of device performance.
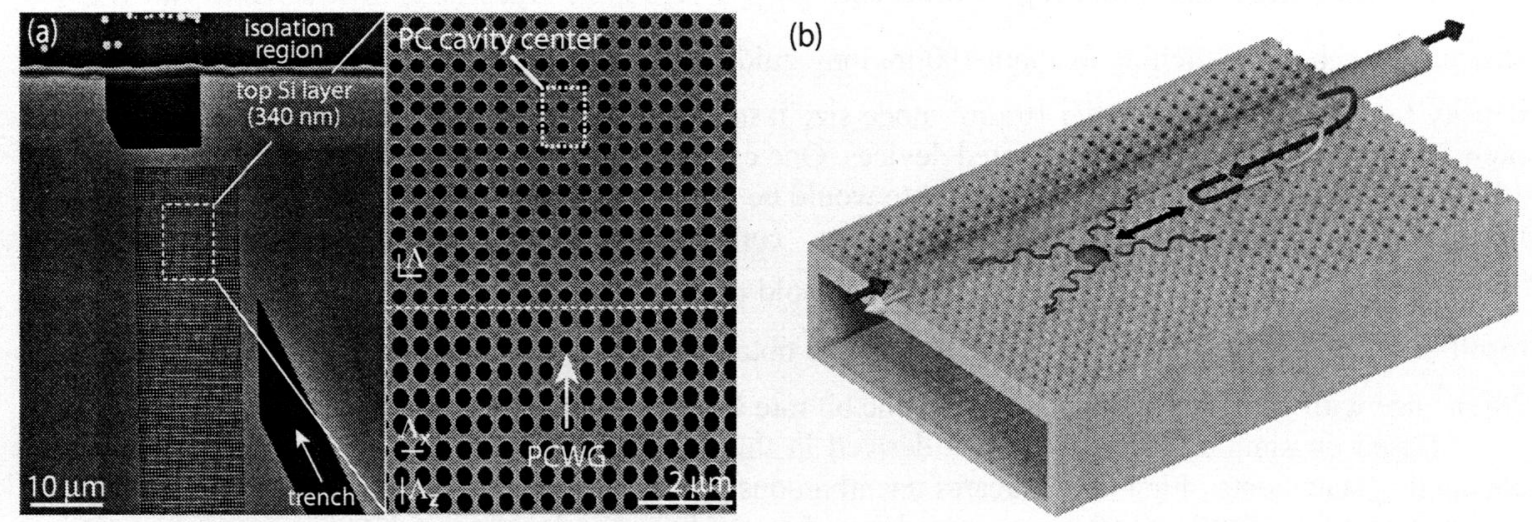

Fig. 1. (a) SEM image of an integrated PC waveguide - PC cavity sample. The PC cavity and PC waveguide have lattice constants $\Lambda \sim 430 \mathrm{~nm}, \Lambda_{x} \sim 430 \mathrm{~nm}$, and $\Lambda_{z} \sim 550 \mathrm{~nm}$. The surrounding silicon material has been removed to form a diagonal trench and isolated mesa structure to enable fiber taper probing. (b) Schematic of the fiber taper - PC waveguide PC cavity coupling scheme.

The photonic crystal structure studied here is shown in Fig. 1(a), and consists of a graded lattice PC cavity [2] integrated with a mode matched PC waveguide [11]. The waveguide is designed to ideally load the cavity; the high-Q cavity mode of interest radiates preferentially into the fundamental guided mode of the PC waveguide. In addition, the waveguide can be evanescently coupled with close to unity efficiency [12] to a fiber taper [13], as illustrated in Fig. 1(b). Light is coupled contra-directionally from the fiber taper into the PC waveguide, and light reflected or radiated by the cavity into the PC waveguide is collected in the backwards propagating fiber taper mode. By studying light which is coupled from the fiber taper into the PC waveguide, reflected by the cavity, and recollected in the backward propagating fiber taper mode, the response of the PC cavity can be measured. Thus, the resulting fiber taper coupled PC waveguide provides an efficient interface between the ultra-small cavity mode and conventional fiber optics. 


\section{QMF3}

Using this technique, a total coupling efficiency of $44 \%$ into a PC cavity mode with a loaded quality factor of $3.8 \times 10^{4}$ from a fiber taper was achieved [10]. Figure 2 shows the resulting cavity response, $R_{o}$, as a function of wavelength, for varying power. $R_{o}$ was obtained by measuring the reflected fiber signal in a $\sim 1 \mu$ m diameter fiber taper aligned $\sim 800 \mathrm{~nm}$ above the PC waveguide in Fig. 1(a), and is normalized by the slowly varying fiber-PC waveguide coupler lineshape. The PC waveguide to taper coupling efficiency was $\sim 73 \%$ in this wavelength range for the device studied here. The sharp dip in reflection, at $\lambda \sim 1589.7 \mathrm{~nm}$, corresponds to resonant excitation of the high-Q PC cavity mode. The cold cavity (low power) linewidth of this resonance is $\delta \lambda \sim 40 \mathrm{pm}$, and the cold cavity resonance contrast, $\Delta R_{o}$, is $60 \%$.

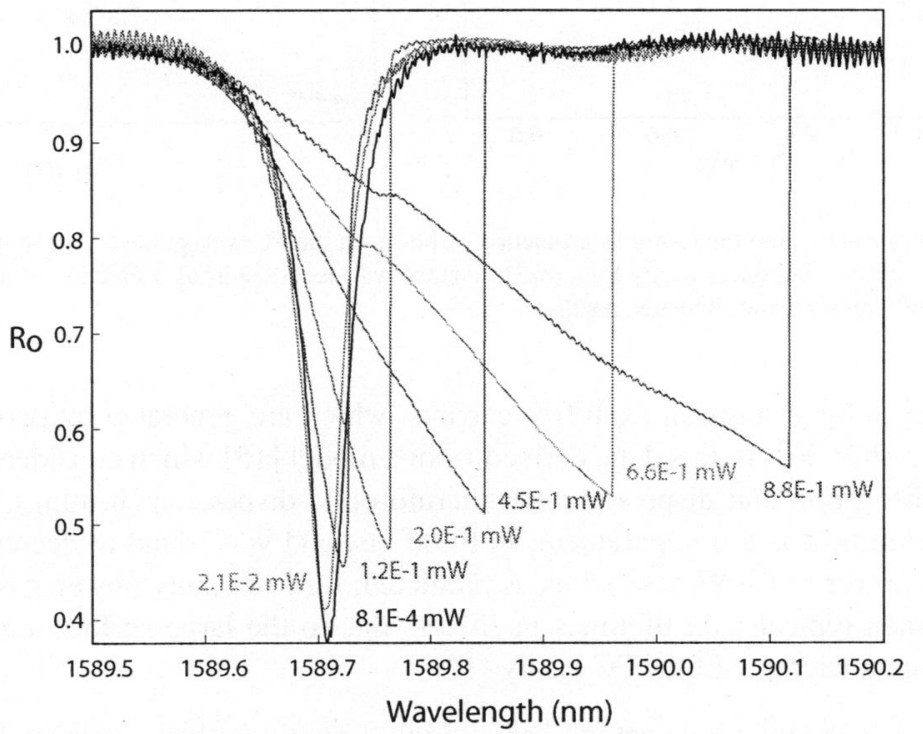

Fig. 2. Measured cavity response as a function of input wavelength, for varying PC waveguide power (taper diameter $d \sim 1$ $\mu \mathrm{m}$, taper height $g=0.80 \mu \mathrm{m})$. The source wavelength is scanned from blue to red in all of the measurements.

To study the nonlinear response of the cavity, the power input to the fiber taper, and consequently the PC waveguide, was varied. Increasing the power results in three readily observable changes in $R_{o}$ : (i) a decrease in the resonance contrast, $\Delta R_{o}$, (ii) a shift $\Delta \omega_{o}$ in the resonance frequency $\omega_{o}$, (iii) broadening and asymmetric distortion of the resonance lineshape, eventually leading to a "snap" in the transmission response characteristic of bistability. As discussed below, these features are due to nonlinear absorption and dispersion in the PC cavity.

The resonance contrast is a measure of the degree of coupling between the cavity and the waveguide, and can be expressed in terms of the intrinsic loss rate of the cavity (due to radiation and absorption for example), $\gamma^{i}$, and the loss rate due to loading from the waveguide, $\gamma^{e}$. In the presence of nonlinear loss, such as two photon and free carrier absorption, $\gamma^{i}$ increases with increasing stored cavity energy, and the coupling parameter, $K=\gamma^{e} / \gamma^{i}$, decreases. For $K<1$ (under-coupled), this results in a decrease in $\Delta R_{o}$ with increasing power, as observed in Fig. 2.

The power dependence of the resonance frequency of the cavity is a result of an intensity dependent cavity refractive index. The Kerr effect, free carrier dispersion, and thermo-optic dispersion, all contribute to shift the material index as the stored cavity energy is increased. The power dependent resonance frequency also gives rises to the asymmetry in the cavity lineshape for increased power, and for a shift $\left|\Delta \omega_{o}\right|>\delta \omega \sqrt{3} / 2$, where $\delta \omega$ is the cavity linewidth, the cavity response is bistable. This bistable response manisfests in a discontinuous "snap" in $R_{o}$ when the source wavelength is scanned through the resonance, as shown in Fig. 2 (wavelength is varried from blue to red here). When the source wavelength is scanned in the opposite direction, the cavity response is hysteretic; i.e. the snap occurs at a different (blue shifted) wavelength.

Fig. 3(a) shows the power dropped into the cavity as a function of the power in the PC waveguide, and Fig. 3(b) shows the shift in the resonance wavelength, $\Delta \lambda_{o}$, as a function of stored cavity energy $U$. Note that for $U>3 \mathrm{fJ}$, corresponding to a dropped cavity power of $>100 \mu \mathrm{W}$, and a fiber taper input power of $>250 \mu \mathrm{W},\left|\Delta \lambda_{o}\right|>\sqrt{3} \delta \lambda / 2 \sim$ $35 \mathrm{pm}$, and the cavity response is bistable. Also, for $U<0.34 \mathrm{fJ}$, the resonance wavelength blue-shifts with increasing power, indicating that free carrier dispersion is the dominant dispersive process for low cavity energy [14]. For larger 

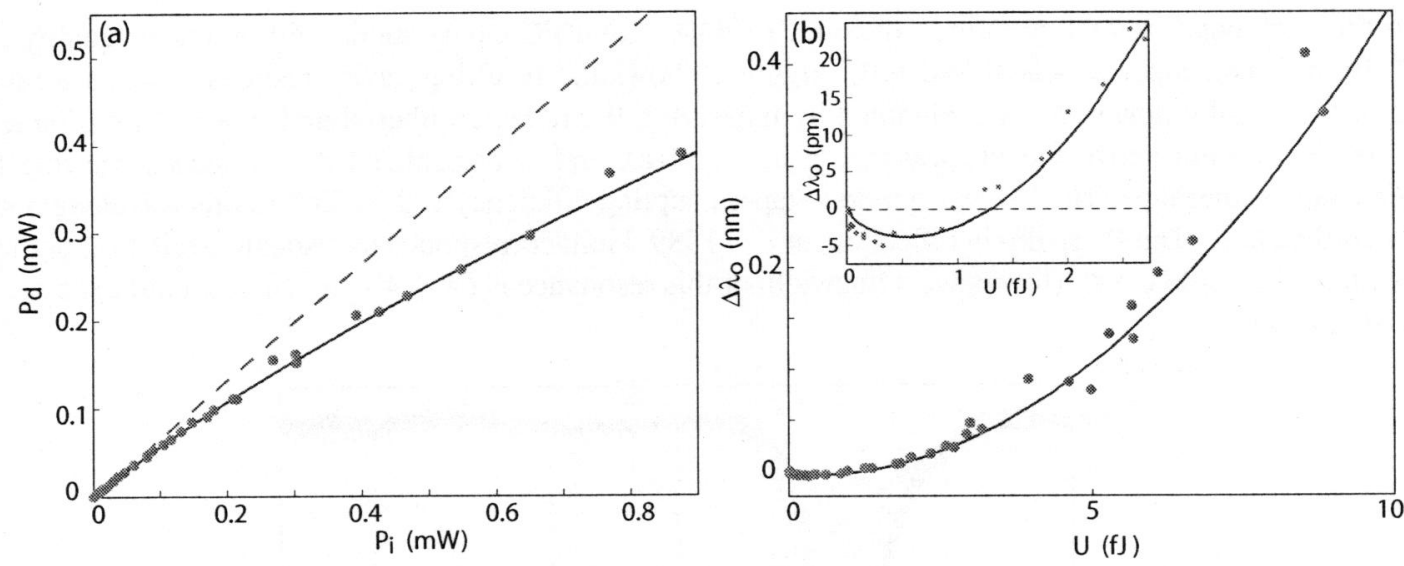

Fig. 3. (a) Power dropped $\left(P_{d}\right)$ into the cavity as a function of power in the PC waveguide $\left(P_{i}\right)$. The dashed line shows the expected result in absence of nonlinear cavity loss. (b) Resonance wavelength shift as a function of internal cavity energy. Solid blue lines in both figures show simulated results.

power, thermal effects driven by absorption from free carriers, which are generated by two photon absorption, cause the cavity resonance to red-shift. Fits to this data, derived from a model [15] which considers the effects of two photon absorption, free carrier absorption and dispersion, and thermo-optic dispersion (heating) in the PC cavity, are also shown. The free carrier lifetime $\tau$ is a free parameter in these fits, and was found to decrease significantly with free carrier density. For cavity power $\sim 1 \mathrm{~mW}, \tau \sim 0.5 \mathrm{~ns}$, is predicted; for low cavity power, $\tau \sim 50-75 \mathrm{~ns}$. These values are significantly smaller than typical bulk lifetimes in silicon, due to the large surface area to volume ratio and the ultra-small mode volume characteristic of the PC cavity [16].

These results confirm that PC microresonators display highly nonlinear behaviour for low input powers, and are promising structures for future application in nonlinear optical switching. In addition, they demonstrate the utility of the fiber taper coupling scheme for performing experiments in PC cavities when optical fidelity is important.

\section{References}

1. Y. Akahane, T. Asano, B.-S. Song, and S. Noda, "High-Q photonic nanocavity in a two-dimensional photonic crystal," Nature 425, $944-947$ (2003).

2. K. Srinivasan, P. E. Barclay, M. Borselli, and O. Painter, "Optical-fiber based measurement of an ultra-small volume high-Q photonic crystal microcavity," Phys. Rev. B p. 081306(R) (2004).

3. A. R. Cowan and J. F. Young, "Optical bistability involving photonic crystal microcavities and Fano line shapes," Phys. Rev. E 68,046606 (2003).

4. M. Soljačić, S. G. Johnson, Y. Fink, and J. D. Joannopoulos, "Optimal bistable switching in nonlinear photonic crystals," Phys. Rev. E 66, 055601 (2002).

5. V. R. Almeida, C. A. Barrios, R. R. Panepucci, and M. Lipson, “All-optical control of light on a silicon chip," Nature (London) 431, 10811084 (2004).

6. V. R. Almeida and M. Lipson, "Optical bistability on a silicon chip," Opt. Lett. 29, 2387-2389 (2004).

7. T. Yoshie, A. Scherer, J. Hendrickson, G. Khitrova, H. Gibbs, G. Rupper, C. Ell, O. Shchekin, and D. Deppe, "Vacuum Rabi splitting with a single quantum dot in a photonic crystal nanocavity," Nature (London) 432, 200-203 (2004).

8. J. Reithmaier, G. Sek, A. Loffler, C. Hofmann, S. Kuhn, S. Reitzenstein, L. Keldysh, V. Kulakovskii, and T. Reinecke, "Strong coupling in a single quantum dot-semiconductor microcavity system," Nature (London) 432, 197-200 (2004).

9. B. Lev, K. Srinivasan, P. E. Barclay, O. Painter, and H. Mabuchi, "Feasibility of detecting single atoms using photonic bandgap cavities," Nanotechnology pp. S556-S561 (2004).

10. P. E. Barclay, K. Srinivasan, and O. Painter, "Optical fiber coupling to planar photonic crystal microcavities," physics/0405064 (2004).

11. P. E. Barclay, K. Srinivasan, and O. Painter, "Design of photonic crystal waveguides for evanescent coupling to optical fiber tapers and integration with high-Q cavities,” J. Opt. Soc. Am. B 20, 2274-2284 (2003).

12. P. E. Barclay, K. Srinivasan, M. Borselli, and O. Painter, "Efficient input and output optical fiber coupling to a photonic crystal waveguide," Opt. Lett. 29, 697-699 (2004).

13. R. P. Kenny, T. A. Birks, and K. P. Oakley, "Control of optical fibre taper shape," IEE Elec. Lett. 27, 1654-1656 (1991).

14. R. A. Soref and B. R. Bennett, "Electrooptical Effects in Silicon," IEEE J. Quan. Elec. 23, 123-129 (1987).

15. P. E. Barclay, K. Srinivasan, and O. Painter, "Nonlinear absorption and dispersion in fiber-taper-coupled photonic crystal microresonators," Submitted (2004).

16. R. Claps, V. Raghunathan, D. Dimitropoulos, and B. Jalali, "Influence of nonlinear absorption on Raman amplification in Silicon waveguides," Opt. Expr. 12, 2774-2780 (2004). 